\title{
The relation between the decomposition of comodules and coalgebras *
}

\author{
Shouchuan Zhang \\ Department of Mathematics, Hunan University \\ Changsha 410082, P.R.China. E-mail:z9491@yahoo.com.cn
}

\begin{abstract}
T. Shudo and H. Miyamito [3] showed that $C$ can be decomposed into a direct sum of its indecomposable subcoalgebras of $C$. Y.H. Xu [5] showed that the decomposition was unique. He also showed that $M$ can uniquely be decomposed into a direct sum of the weak-closed indecomposable subcomodules of $M$ (we call the decomposition the weak-closed indecomposable decomposition ) in [6]. In this paper, we give the relation between the two decomposition. We show that if $M$ is a full, $W$-relational hereditary $C$-comodule, then the following conclusions hold:

(1) $M$ is indecomposable iff $C$ is indecomposable;

(2) $M$ is relative-irreducible iff $C$ is irreducible;

(3) $M$ can be decomposed into a direct sum of its weak-closed relative-irreducible subcomodules iff $C$ can be decomposed into a direct sum of its irreducible subcoalgebras.

We also obtain the relation between coradical of $C$ - comodule $M$ and radical of algebra $C(M)^{*}$
\end{abstract}

\section{Introduction and Preliminaries}

The decomposition of coalgebras and comodules is an important subject in study of Hopf algebras. T. Shudo and H. Miyamito [3] showed that $C$ can be decomposed into a direct sum of its indecomposable subcoalgebras of $C$. Y.H. Xu [5] showed that the decomposition was unique. He also showed that $M$ can uniquely be decomposed into a direct sum of the weak-closed indecomposable subcomodules of $M$ (we call the decomposition the weakclosed indecomposable decomposition ) in [6]. In this paper, we give the relation between

*This work was supported by the National Natural Science Foundation 
the two decomposition. We show that if $M$ is a full, $W$-relational hereditary $C$-comodule, then the following conclusions hold:

(1) $M$ is indecomposable iff $C$ is indecomposable;

(2) $M$ is relative-irreducible iff $C$ is irreducible;

(3) $M$ can be decomposed into a direct sum of its weak-closed relative-irreducible subcomodules iff $C$ can be decomposed into a direct sum of its irreducible subcoalgebras. We also obtain the relation between coradical of $C$ - comodule $M$ and radical of algebra $C(M)^{*}$

Let $k$ be a field, $M$ be a $C$-comodule, $N$ be a subcomodule of $M, E$ be a subcoalgebras of $C$ and $P$ be an ideal of $C^{*}$. As in [6], we define:

$$
\begin{aligned}
& E^{\perp C^{*}}=\left\{f \in C^{*} \mid f(E)=0\right\} . \\
& P^{\perp C}=\{c \in C \mid P(c)=0\} . \\
& N^{\perp C^{*}}=\left\{f \in C^{*} \mid f \cdot N=0\right\} . \\
& P^{\perp M}=\{x \in M \mid P \cdot x=0\} .
\end{aligned}
$$

Let $\left\langle N>\right.$ denote $N^{\perp C^{*} \perp M}$. $\langle N>$ is called the closure of $N$. If $\langle N\rangle=N$, then $N$ is called closed. If $N=C^{*} x$, then $\langle N\rangle$ is denoted by $\langle x\rangle$. If $\langle x\rangle \subseteq N$ for any $x \in N$, then $N$ is called weak-closed. It is clear that any closed subcomodule is weak-closed. If $\rho(N) \subseteq N \otimes E$, then $N$ is called an $E$-subcomodule of $M$. Let

$$
M_{E}=\sum\{N \mid N \text { is a subcomodule of } M \text { and } \rho(N) \subseteq N \otimes E\}
$$

We call $M_{E}$ a component of $M$ over $E$. If $M_{E}$ is some component of $M$ and $M_{F} \subseteq M_{E}$ always implies $M_{F}=M_{E}$ for any non-zero component $M_{F}$, then $M_{E}$ is called a minimal component of $M$.

Let $\left\{m_{\lambda} \mid \lambda \in \Lambda\right\}$ be the basis of $M$ and $C(M)$ denote the subspace of $C$ spanned by $W(M)=\left\{c \in C \mid\right.$ there exists an $m \in M$ with $\rho(m)=\sum m_{\lambda} \otimes c_{\lambda}$ such that $c_{\lambda_{0}}=$ c for some $\left.\lambda_{0} \in \Lambda\right\}$.

E. Abe in [1, P129] checked that $C(M)$ is a subcoalgebra of $C$. It is easy to know that if $E$ is subcoalgebra of $C$ and $\rho(M) \subseteq M \otimes E$, then $C(M) \subseteq E$. If $C(M)=C$, then $M$ is called a full $C$-comodule. If $D$ is a simple subcoalgbra of $C$ and $M_{D} \neq 0$ or $D=0$, then $D$ is called faithful to $M$. If every simple subcoalgbra of $C$ is faithful to $M$, then $M$ is called a component faithfulness $C$-comodule.

Let $X$ and $Y$ be subspaces of coalgebra $C$. Define $X \wedge Y$ to be the kernel of the composite

$$
C \stackrel{\Delta}{\rightarrow} C \otimes C \rightarrow C / X \otimes C / Y
$$

$X \wedge Y$ is called a wedge of $X$ and $Y$. 


\section{The relation between the decomposition of comod- ules and coalgebras}

Lemma 1.1 Let $N$ and $L$ be subcomodules of $M$. Let $D$ and $E$ be subcoalgebras of $C$. Then

(1) $N^{\perp C^{*}}=(C(N))^{\perp C^{*}}$;

(2) $M_{E}=M_{C\left(M_{E}\right)}$;

(3) $N$ is closed iff there exists a subcoalgebra $E$ such that $N=M_{E}$;

(4) $M_{D} \neq 0$ iff $D^{\perp C^{*} \perp M} \neq 0$;

(5) If $D \cap C(M)=0$, then $M_{D}=0$;

(6) If $D$ is a simple subcoalgebra of $C$, then $C\left(M_{D}\right)=\left\{\begin{array}{ll}D & \text { if } M_{D} \neq 0 \\ 0 & \text { if } M_{D}=0\end{array}\right.$;

(7) If $D \cap E=0$, then $M_{D} \cap M_{E}=0$;

(8) If $D$ and $E$ are simple subcoalgebras and $M_{D} \cap M_{E}=0$ with $M_{D} \neq 0$ or $M_{E} \neq 0$, then $D \cap E=0$.

(9) If $M_{E}$ is the minimal component of $M$ and $0 \neq N \subseteq M_{E}$, then $C\left(M_{E}\right)=C(N)$.

Proof. (1) Let $\left\{m_{\lambda} \mid \lambda \in \Lambda\right\}$ be a basis of $N$. For any $f \in N^{\perp C^{*}}$, if $c \in W(N)$, then there exists $m \in N$ with $\rho(m)=\sum_{\lambda \in \Lambda} m_{\lambda} \otimes c_{\lambda}$ such that $c_{\lambda_{0}}=c$ for some $\lambda_{0} \in \Lambda$. Since $f \cdot m=\sum m_{\lambda} f\left(c_{\lambda}\right)=0, f\left(c_{\lambda}\right)=0$ for any $\lambda \in \Lambda$. Obviously, $f(c)=f\left(c_{\lambda_{0}}\right)=0$. Considering that $C(N)$ is the space spanned by $W(N)$, we have $f \in C(N)^{\perp C^{*}}$. Conversely, if $f \in C(N)^{\perp C^{*}}$, then $f \cdot m=\sum m_{\lambda} f\left(c_{\lambda}\right)=0$ for any $m \in N$, i.e. $f \in N^{\perp C^{*}}$. This shows that $N^{\perp C^{*}}=C(N)^{\perp C^{*}}$.

(2) Since $M_{E}$ is an $E$-subcomodule of $M, C\left(M_{E}\right) \subseteq E$ and $M_{C\left(M_{E}\right)} \subseteq M_{E}$. Since $M_{E}$ is a $C\left(M_{E}\right)$-subcomodule, $M_{E} \subseteq M_{C\left(M_{E}\right)}$.

(3) If $N$ is closed, then $N^{\perp C^{*} \perp M}=N$. Let $E=C(N)$. Obviously, $N \subseteq M_{E}$. By Lemma 1.1(1), $N^{\perp C^{\star}} \cdot M_{E}=E^{\perp C^{\star}} \cdot M_{E}=0$. Thus $M_{E} \subseteq N^{\perp C^{\star} \perp M}$ and $M_{E} \subseteq N$. This shows that $M_{E}=N$. Conversely, if $M_{E}=N$, obviously, $\left(M_{E}\right) \subseteq\left(M_{E}\right)^{\perp C^{*} \perp M}$. Thus it is sufficient to show that

$$
<M_{E}>\subseteq M_{E}
$$

Let $L=\left(M_{E}\right)^{\perp C^{*} \perp M}=<M_{E}>$. We see that $C(L)^{\perp C^{*}}=L^{\perp C^{*}}=\left(M_{E}\right)^{\perp C^{*} \perp M \perp C^{*}}=$ $\left(M_{E}\right)^{\perp C^{*}}=C\left(M_{E}\right)^{\perp C^{*}}$. Thus $C(L)=C\left(M_{E}\right)$ and $L \subseteq M_{C(L)}=M_{C\left(M_{E}\right)}=M_{E}$.

(4) If $D^{\perp C^{*} \perp M} \neq 0$, let $\left\{m_{\lambda} \mid \lambda \in \Lambda\right\}$ be a basis of $D^{\perp C^{*} \perp M}$ and $\rho(x)=\sum m_{\lambda} \otimes d_{\lambda}$ for any $x \in D^{\perp C^{*} \perp M}$. Since $D^{\perp C^{*}} \cdot x=\sum_{\lambda} m_{\lambda} D^{\perp C^{*}}\left(d_{\lambda}\right)=0, d_{\lambda} \in D^{\perp C^{*} \perp C}=D$ for any $\lambda \in \Lambda$, which implies that $D^{\perp C^{*} \perp M}$ is a $D$-subcomodule. Therefore $0 \neq D^{\perp C^{*} \perp M} \subseteq M_{D}$, i.e. $M_{D} \neq 0$. Conversely, if $M_{D} \neq 0$, then we have that

$$
\begin{aligned}
0 \neq M_{D} & \subseteq\left(M_{D}\right)^{\perp C^{*} \perp M} \\
& =C\left(M_{D}\right)^{\perp C^{*} \perp M} \quad(\text { by part }(1))
\end{aligned}
$$




$$
\subseteq D^{\perp C^{*} \perp M} .
$$

(5) Since $M_{D} \subseteq M, C\left(M_{D}\right) \subseteq C(M)$. Obviously, $C\left(M_{D}\right) \subseteq D$. Thus $C\left(M_{D}\right) \subseteq$ $C(M) \cap D=0$, which implies that $M_{D}=0$.

(6) If $M_{D}=0$, then $C\left(M_{D}\right)=0$. If $M_{D} \neq 0$, then $0 \neq C\left(M_{D}\right) \subseteq D$. Since $D$ is a simple subcoalgebra, $C\left(M_{D}\right)=D$.

(7) If $x \in M_{D} \cap M_{E}$, then $\rho(x) \in\left(M_{D} \otimes D\right) \cap\left(M_{E} \otimes E\right)$, and

$$
\rho(x)=\sum_{i=1}^{n} x_{i} \otimes d_{i}=\sum_{j=1}^{m} y_{j} \otimes e_{j},
$$

where $x_{i}, \cdots, x_{n}$ is linearly independent and $d_{i} \in D$ and $e_{j} \in E$. Let $f_{l} \in M^{*}$ with $f_{l}\left(x_{i}\right)=\delta_{l i}$ for $i, l=1,2, \cdots n$. Let $f_{l} \otimes i d$ act on equation (1). We have that $d_{l}=$ $\sum_{j=1}^{m} f_{l}\left(y_{j}\right) e_{j} \in E$, which implies $d_{l} \in D \cap E=0$ and $d_{l}=0$ for $l=1, \cdots, n$. Therefore $M_{D} \cap M_{E}=0$.

(8) If $D \cap E \neq 0$, then $D=E$. Thus $M_{D}=M_{E}$ and $M_{D} \cap M_{E}=M_{E}=M_{D}=0$. We get a contradiction. Therefore $D \cap E=0$.

(9) Obviously, $C(N) \subseteq C\left(M_{E}\right)$. Conversely,since $0 \neq N \subseteq M_{C(N)} \subseteq M_{C\left(M_{E}\right)}=$ $M_{E}$ and $M_{E}$ is a minimal component, $M_{C(N)}=M_{E}$. By the definition of component, $C\left(M_{E}\right) \subseteq C(N)$. Thus $C(N)=C\left(M_{E}\right)$.

Proposition 1.2 If $E$ is a subcoalgebra of $C$, then the following conditions are equivalent.

(1) $M_{E}$ is a minimal component of $M$.

(2) $C\left(M_{E}\right)$ is a simple subcoalgebra of $C$.

(3) $M_{E}$ is a minimal closed subcomodule of $M$.

Proof. It is easy to check that $M_{E}=0$ iff $C\left(M_{E}\right)=0$. Thus (1), (2) and (3) are equivalent when $C\left(M_{E}\right)=0$, We now assume that $M_{E} \neq 0$.

$(1) \Longrightarrow(2)$ Since $0 \neq M_{E}$, there exists a non-zero finite dimensional simple subcomodule $N$ of $M$ such that $N \subseteq M_{E}$. By [6, Lemma 1.1], $N$ is a simple $C^{*}$-submodule of $M$. Since $M_{E}$ is a minimal component, $C(N)=C\left(M_{E}\right)$ by Lemma 1.1 (9). Let $D=C(N)=C\left(M_{E}\right)$. By Lemma 1.1(1), $(0: N)_{C^{*}}=N^{\perp C^{*}}=C(N)^{\perp C^{*}}=D^{\perp C^{*}}$. Thus $N$ is a faithful simple $C^{*} / D^{\perp C^{*}}$-module, and so $C^{*} / D^{\perp C^{*}}$ is a simple algebra.It is clear that $D$ is a simple subcoalgebra of $C$.

(2) $\Longrightarrow$ (3) If $0 \neq N \subseteq M_{E}$ and $N$ is a closed subcomodule of $M$, then by Lemma 1.1(3) there exists a subcoalgebra $F$ of $C$ such that $N=M_{F}$. Since $0 \neq C(N)=C\left(M_{F}\right) \subseteq$ $C\left(M_{E}\right)$ and $C\left(M_{E}\right)$ is simple, $C\left(M_{F}\right)=C\left(M_{E}\right)$. By Lemma 1.1(2), $N=M_{F}=M_{C\left(M_{F}\right)}=$ $M_{C\left(M_{E}\right)}=M_{E}$, which implies that $M_{E}$ is a minimal closed subcomodule.

(3) $\Longrightarrow(1)$, If $0 \neq M_{F} \subseteq M_{E}$, then $M_{F}$ is a closed subcomodule by Lemma 1.1(3) and $M_{E}=M_{F}$, i.e. $M_{E}$ is a minimal component. 
This completes the proof.

Let

$\mathcal{C}_{0}=\{D \mid D$ is a simple subcoalgebra of $C\}$;

$\mathcal{C}(M)_{0}=\{D \mid D$ is a simple subcoalgebra of $C$ and $D \subseteq C(M)\}$;

$\mathcal{M}_{0}=\{N \mid N$ is a minimal closed subcomodule of $M\}$;

$\mathcal{C}(M)_{1}=\{D \mid D$ is a faithful simple subcoalgebra of $C$ to $M\}$;

$C_{0}=\sum\{D \mid D$ is a simple subcoalgebra of $C\}$;

$M_{0}=\sum\{N \mid N$ is a minimal closed subcomodule of $M\}$;

$C_{0}$ and $M_{0}$ are called the coradical of coalgebra $C$ and the coradical of comodule $M$ respectively. If $M_{0}=M$, then $\mathrm{M}$ is called cosemisimple. By Lemma 1.1(5),

$\mathcal{C}(M)_{1} \subseteq \mathcal{C}(M)_{0}$

Theorem 1.3 $\psi\left\{\begin{array}{l}\mathcal{C}(M)_{1} \longrightarrow \mathcal{M}_{0} \\ D \longmapsto M_{D}\end{array} \quad\right.$ is bijective.

Proof. By Proposition 1.2, $\psi$ is a map. Let $D$ and $E \in \mathbf{C}(M)_{1}$ and $\psi(D)=\psi(E)$, i.e. $M_{D}=M_{E}$. By Lemma $1.1(6)$, we have that $D=C\left(D_{D}\right)=C\left(M_{E}\right)=E$. If $N \in \mathcal{M}_{0}$, then $N=M_{C(N)}$ and $C(N)$ is a simple subcoalgebra by Lemma 1.1(3) and Proposition 1.2. Thus $\psi(C(N))=N$, which implies that $\psi$ is surjective.

In [6] and [5], Xu defined the equivalence relation for coalgebra and for comodule as follows:

Definition 1.4 We say that $D \sim E$ for $D$ and $E \in \mathcal{C}_{0}$ iff for any pair of subclasses $\mathcal{C}_{1}$ and $\mathcal{C}_{2}$ of $\mathcal{C}_{0}$ with $D \in \mathcal{C}_{1}$ and $E \in \mathcal{C}_{2}$ such that $\mathcal{C}_{1} \cup \mathcal{C}_{2}=\mathcal{C}_{0}$ and $\mathcal{C}_{1} \cap \mathcal{C}_{2}=\emptyset$, there exist elements $D_{1} \in \mathcal{C}_{1}$ and $E_{1} \in \mathcal{C}_{2}$ such that $D_{1} \wedge E_{1} \neq E_{1} \wedge D_{1}$. Let $[D]$ denote the equivalence class which contains $D$.

We say that $N \sim L$ for $N$ and $L \in \mathcal{M}_{0}$ iff for any pair of subclasses $\mathcal{M}_{1}$ and $\mathcal{M}_{2}$ of $\mathcal{M}_{0}$ with $N \in \mathcal{M}_{1}$ and $L \in \mathcal{M}_{2}$ such that $\mathcal{M}_{1} \cup \mathcal{M}_{2}=\mathcal{M}_{0}$ and $\mathcal{M}_{1} \cap \mathcal{M}_{2}=\emptyset$, there exist elements $N_{1} \in \mathcal{M}_{1}$ and $L_{1} \in \mathcal{M}_{2}$ such that $N_{1} \wedge L_{1} \neq L_{1} \wedge N_{1}$. Let $[N]$ denote the equivalence class which contains $N$.

Definition 1.5 If $D \wedge E=E \wedge D$ for any simple subcoalgebras $D$ and $E$ of $C$, then $C$ is called $\pi$-commutative. If $N \wedge L=L \wedge N$ for any minimal closed subcomodules $N$ and $L$ of $M$, then $M$ is called $\pi$-commutative.

Obviously, every cocommutative coalgebra is $\pi$-commutative. By [6, Theorem 3.8 and Theorem 4.18], $M$ is $\pi$-commutative iff $M$ can be decomposed into a direct sum of the weak-closed relative-irreducible subcomodules of $M$ iff every equivalence class of $M$ contains only one element. By [5], $C$ is $\pi$-commutative iff $C$ can be decomposed into a direct sum of irreducible subcoalgebras of $C$ iff equivalence every class of $C$ contains only one element. 
Lemma 1.6 Let D, E and $F$ be subcoalgebras of $C . N, L$ and $T$ be subcomodules of M. Then

(1) $M_{D} \wedge M_{F}=M_{C\left(M_{D}\right) \wedge C\left(M_{F}\right)} \subseteq M_{D \wedge F}$;

(2) If $D$ and $E$ are faithful simple subcoalgebras of $C$ to $M$, then $M_{D} \wedge M_{E}=M_{D \wedge F}$;

(3) $M_{D+E} \supseteq M_{D}+M_{E}$;

(4) If $F=\sum\left\{D_{\alpha} \mid \alpha \in \Omega\right\}$ and $\left\{D_{\alpha} \mid \alpha \in \Omega\right\} \subseteq \mathcal{C}_{0}$, then $M_{F}=\sum\left\{M_{D_{\alpha}} \mid \alpha \in \Omega\right\}$.In particular, $M_{C_{0}}=M_{0}$.

(5) $(N+L) \wedge T \supseteq N \wedge T+L \wedge T$;

(6) $(D+E) \wedge F \supseteq D \wedge F+E \wedge F$.

Proof. (1) We see that

$$
\begin{aligned}
M_{D} \wedge M_{E} & =\rho^{-1}\left(M \otimes\left(M_{D}\right)^{\perp C^{*} \perp C} \wedge\left(M_{E}\right)^{\perp C^{*} \perp C}\right) \quad(\text { by }[6, \text { Proposition 2.2(1)] }) \\
& =\rho^{-1}\left(M \otimes C\left(M_{D}\right) \wedge C\left(M_{E}\right)\right) \quad(\text { by Lemma 1.1(1)). }
\end{aligned}
$$

By the definition of component, subcomodule $M_{D} \wedge M_{E} \subseteq M_{C\left(M_{D}\right) \wedge C\left(M_{E}\right)}$. It follows from the equation above that $M_{C\left(M_{D}\right) \wedge C\left(M_{E}\right)} \subseteq M_{D} \wedge M_{E}$. Thus

$$
M_{D} \wedge M_{E}=M_{C\left(M_{D}\right) \wedge C\left(M_{E}\right)}
$$

and

$$
M_{C\left(M_{D}\right) \wedge C\left(M_{E}\right)} \subseteq M_{D \wedge E} .
$$

(2) Since $D$ and $E$ are faithful simple subcoalgebras of $C$ to $M, C\left(M_{D}\right)=D$ and $C\left(M_{E}\right)=E$ by Lemma 1.1(6). By Lemma 1.6(1), $M_{D} \wedge M_{E}=M_{D \wedge E}$.

(3) It is trivial.

(4) Obviously $M_{F} \supseteq \sum\left\{M_{D_{\alpha}} \mid \alpha \in \Omega\right\}$. Conversely, let $N=M_{F}$. Obviously $N$ is an $F$-comodule. For any $x \in N$, let $L=C^{*} x$. it is clear that $L$ is a finite dimensional comodule over $F$. By [4, Lemma 14.0.1], $L$ is a completely reducible module over $F^{*}$. Thus $L$ can be decomposed into a direct sum of simple $F^{*}$-submodules:

$$
L=L_{1} \oplus L_{2} \oplus \cdots \oplus L_{n}
$$

where $L_{i}$ is a simple $F^{*}$-submodule. By [6, Proposition 1.16], $\left\langle L_{i}\right\rangle=\left(L_{i}\right)^{\perp F^{*} \perp N}$ is a minimal closed $F$-subcomodule of $N$. By Theorem 1.3, there exists a simple subcoalgebra $D_{\alpha_{i}}$ of $F$ such that $\left\langle L_{i}\right\rangle=N_{D_{\alpha_{i}}}$. Obviously, $N_{D_{\alpha_{i}}} \subseteq M_{D_{\alpha_{i}}}$. Thus $L \subseteq \sum_{i=1}^{n}<L_{i}>=$ $\sum_{i=1}^{n} N_{D_{\alpha_{i}}} \subseteq \sum\left\{M_{D_{\alpha}} \mid \alpha \in \Omega\right\}$. Therefore $M_{F}=\sum\left\{M_{D_{\alpha}} \mid \alpha \in \Omega\right\}$. If $C_{0}=F=\sum\{D \mid$ $\left.D \in \mathcal{C}_{0}\right\}$, then $M_{C_{0}}=M_{F}=\sum\left\{M_{D} \mid D \in \mathcal{C}_{0}\right\}=M_{0}$ by Theorem 1.3,

(5) and (6) are trivial. $\square$

Lemma 1.7 Let $N$ be a subcomodule of $M$, and let $D, E$ and $F$ be simple subcoalgebras of $C$. Then 
(1) $D \sim 0$ iff $D=0 ; \quad M_{D} \sim 0$ iff $M_{D}=0$;

We called the equivalence class which contains zero a zero equivalence class.

(2) If $D$ and $E$ are faithful to $M$ and $M_{D} \sim M_{E}$, then $D \sim E$;

(3) $\left[M_{D}\right] \subseteq\left\{M_{E} \mid E \in[D]\right\}$;

(4) If $D$ is faithful to $M$, then $\left[M_{D}\right] \subseteq\left\{M_{E} \mid E \in[D]\right.$ and $E$ is faithful to $\left.M\right\}$.

Proof. (1) If $D \sim 0$ and $D \neq 0$, let $\mathcal{C}_{1}=\{0\}$ and $\mathcal{C}_{2}=\left\{F \mid F \neq 0, F \in \mathcal{C}_{0}\right\}$. Thus $\mathcal{C}_{1} \cup \mathcal{C}_{2}=\mathcal{C}_{0}$ and $\mathcal{C}_{1} \cap \mathcal{C}_{2}=\emptyset$ with $0 \in \mathcal{C}_{1}$ and $D \in \mathcal{C}_{2}$. But for any $D_{1} \in \mathcal{C}_{1}$ and $E_{1} \in \mathcal{C}_{2}$, since $D_{1}=0, D_{1} \wedge E_{1}=E_{1} \wedge D_{1}=E_{1}$. We get a contradiction. Thus $D=0$. Conversely, if $D=0$, obviously $D \sim 0$. Similarly, we can show that $M_{D} \sim 0$ iff $M_{D}=0$.

(2) For any pair of subclasses $\mathcal{C}_{1}$ and $\mathcal{C}_{2}$ of $\mathcal{C}_{0}$, if $\mathcal{C}_{1} \cap \mathcal{C}_{2}=\emptyset$ and $\mathcal{C}_{1} \cup \mathcal{C}_{2}=\mathcal{C}_{0}$ with $D \in \mathcal{C}_{1}$ and $E \in \mathcal{C}_{2}$, let $\mathcal{M}_{1}=\left\{M_{F} \mid F \in \mathcal{C}_{1}\right.$ and $F$ is faithful to $\left.M\right\}$ and $\mathcal{M}_{2}=$ $\left\{M_{F} \mid F \in \mathcal{C}_{2}\right.$ and $F$ is faithful to $\left.M\right\}$. By Theorem 1.3, $\mathcal{M}_{0}=\mathcal{M}_{1} \cup \mathcal{M}_{2}$ and $\mathcal{M}_{1} \cap \mathcal{M}_{2}=\emptyset$. Obviously, $M_{D} \in \mathcal{M}_{1}$ and $M_{E} \in \mathcal{M}_{2}$. Since $M_{D} \sim M_{E}$, there exist $M_{D_{1}} \in \mathcal{M}_{1}$ and $M_{E_{1}} \in \mathcal{M}_{2}$ such that $M_{D_{1}} \wedge M_{E_{1}} \neq M_{E_{1}} \wedge M_{D_{1}}$, where $D_{1} \in \mathcal{C}_{1}$ and $E_{1} \in \mathcal{C}_{2}$. By Lemma 1.6(2), $M_{D_{1} \wedge E_{1}} \neq M_{E_{1} \wedge D_{1}}$. Thus $D_{1} \wedge E_{1} \neq E_{1} \wedge D_{1}$. Obviously $D$ and $D_{1} \in \mathcal{C}_{1}$. Meantime $E$ and $E_{1} \in \mathcal{C}_{2}$. By Definition 1.4, $D \sim E$.

(3) Obviously, $M_{F} \sim M_{D}$ for any $M_{F} \in\left[M_{D}\right]$. If $M_{D} \neq 0$, then $M_{F} \neq 0$ by Lemma 1.7 (1), By Lemma 1.7(2), $F \sim D$. Thus $M_{F} \in\left\{M_{E} \mid E \in[D]\right\}$. If $M_{D}=0$, by Lemma 1.7(1), $M_{F}=0=M_{D} \in\left\{M_{E} \mid E \in[D]\right\}$.

(4) If $M_{F} \in\left[M_{D}\right]$, then $M_{D} \sim M_{F}$. If $D=0$, then $M_{D}=0$. By Lemma 1.7(1), $M_{F}=0$. Thus $M_{F}=M_{D}=0 \in\left\{M_{E} \mid E \in[D]\right.$ and $E$ is faithful to $\left.M\right\}$. If $D \neq 0$, we have that $M_{D} \neq 0$ since $D$ is faithful to $M$. By Lemma 1.7(1), $M_{F} \neq 0$. By Lemma 1.7(3), $M_{F} \in\left\{M_{E} \mid E \in[D]\right.$ and $E$ is faithful to $\left.M\right\}$.

Theorem 1.8 Let $\left\{\mathcal{E}_{\alpha} \mid \alpha \in \bar{\Omega}\right\}$ be all of the equivalence classes of $C$. and $E_{\alpha}=$ $\sum\left\{E \mid E \in \mathcal{E}_{\alpha}\right\}$. Then

(1) For any $\alpha \in \bar{\Omega}$, there exists a set $I_{\alpha}$ and subclasses $\mathcal{E}(\alpha, i) \subseteq \mathcal{E}_{\alpha}$ such that $\cup\left\{\mathcal{E}(\alpha, i) \mid i \in I_{\alpha}\right\}=\mathcal{E}_{\alpha}$ and $\left\{M_{\mathcal{E}(\alpha, i)} \mid \alpha \in \bar{\Omega}, i \in I_{\alpha}\right\}$

is the set of the equivalence classes of $M$ (they are distinct except for zero equivalence class $)$, where $M_{\mathcal{E}(\alpha, i)}$ denotes $\left\{M_{D} \mid D \in \mathcal{E}(\alpha, i)\right\}$.

(2) If $M$ is a component faithfulness $C$-comodule, then $\left\{M_{\mathcal{E}(\alpha, i)} \mid \alpha \in \bar{\Omega}, i \in I_{\alpha}\right\}$ is the set of the distinct equivalence classes of $M$.

(3)

$$
M=\sum_{\alpha \in \bar{\Omega}} \oplus M_{\left(E_{\alpha}\right)}(\infty)=\sum_{\alpha \in \bar{\Omega}} \sum_{i \in I_{\alpha}} \oplus\left(M_{E(\alpha, i)}\right)^{(\infty)}=\sum_{\alpha \in \bar{\Omega}} \oplus\left(M_{E_{\alpha}}\right)^{(\infty)}
$$

and for any $\alpha \in \bar{\Omega}$,

$$
M_{\left(E_{\alpha}\right)(\infty)}=\sum_{i \in I_{\alpha}}\left(M_{E(\alpha, i)}\right)^{(\infty)}=\left(M_{E_{\alpha}}\right)^{(\infty)}
$$


where $E(\alpha, i)=\sum\{E \mid E \in \mathcal{E}(\alpha, i)\}$.

Proof. (1) By Theorem 1.3 and Lemma 1.7(3) we can immediately get part (1).

(2) If $M$ is a component faithfulness $C$-comodule, then $\mathcal{C}(M)_{1}=\mathcal{C}(M)_{0}=\mathcal{C}_{0}$. It follows from Theorem 1.3 and part (1) that $\left\{M_{\mathcal{E}(\alpha, i)} \mid \alpha \in \bar{\Omega}, i \in I_{\alpha}\right\}$ consists of all the distinct equivalence classes of $M$.

(3) We see that

$M=M_{C}=M_{\sum\left\{\left(E_{\alpha}\right)^{(\infty)} \mid \alpha \in \bar{\Omega}\right\}}$ (by [5])

$\supseteq \sum_{\alpha \in \bar{\Omega}} M_{\left(E_{\alpha}\right)}(\infty)$ (by Lemma 1.6(3))

$\supseteq \sum_{\alpha \in \bar{\Omega}} \sum_{n=0}^{\infty} M_{\wedge^{n+1} E_{\alpha}}$ ( by Lemma 1.6(3))

$\supseteq \sum_{\alpha \in \bar{\Omega}} \sum_{n=0}^{\infty} \wedge^{n+1} M_{E_{\alpha}}$ (by Lemma 1.6(1))

$=\sum_{\alpha \in \bar{\Omega}} \sum_{n=0}^{\infty} \wedge^{n+1} M_{\sum_{i \in I_{\alpha}} E(\alpha, i)}$ (by Theorem 1.8(1))

$\supseteq \sum_{\alpha \in \bar{\Omega}} \sum_{n=0}^{\infty} \wedge^{n+1} \sum_{i \in I_{\alpha}} M_{E(\alpha, i)}$ (by Lemma 1.6(3))

$\supseteq \sum_{\alpha \in \bar{\Omega}} \sum_{n=0}^{\infty} \sum_{i \in I_{\alpha}} \wedge^{n+1} M_{E(\alpha, i)}$ ( by Lemma 1.6(5)(6))

$=\sum_{\alpha \in \bar{\Omega}} \sum_{i \in I_{\alpha}} \sum_{n=0}^{\infty} \wedge^{n+1} M_{E(\alpha, i)}$

$=\sum_{\alpha \in \bar{\Omega}} \sum_{i \in I_{\alpha}}\left(M_{E(\alpha, i)}\right)^{(\infty)}$

$=M($ by $[6,(4.10)$ in Theorem4.15] and Lemma 1.6(4) and part (1)).

Thus

$$
M=\sum_{\alpha \in \bar{\Omega}} M_{\left(E_{\alpha}\right)(\infty)}=\sum_{\alpha \in \bar{\Omega}} \sum_{i \in I_{\alpha}}\left(M_{E(\alpha, i)}\right)^{(\infty)}
$$

and

$$
M=\sum_{\alpha \in \bar{\Omega}} \sum_{i \in I_{\alpha}} \oplus\left(M_{E(\alpha, i)}\right)^{(\infty)}
$$

by $[6$, Theorem 4.15$]$ and part (1). We see that

$$
\begin{aligned}
M_{E(\alpha, i)} \wedge M_{E(\alpha, i)} & \subseteq M_{\left(E_{\alpha}\right)^{(\infty)}} \wedge M_{\left(E_{\alpha}\right)^{(\infty)}} \\
& \subseteq M_{\left(E_{\alpha}\right)^{(\infty)} \wedge\left(E_{\alpha}\right)^{(\infty)}} \text { (by Lemma 1.6(1)) } \\
& =M_{\left(E_{\alpha}\right)^{(\infty)}} \text { (by [2, Proposition 2.1.1]) }
\end{aligned}
$$

Thus

$$
M_{\left(E_{\alpha}\right)(\infty)} \supseteq\left(M_{E(\alpha, i)}\right)^{(\infty)}
$$

for any $i \in I_{\alpha}$ and for any $\alpha \in \bar{\Omega}$, and

$$
M_{\left(E_{\alpha}\right)(\infty)} \supseteq \sum_{i \in I_{\alpha}}\left(M_{E(\alpha, i)}\right)^{(\infty)}
$$

If $M_{\left(E_{\alpha}\right)(\infty)} \cap \sum_{\beta \in \bar{\Omega}, \beta \neq \alpha} M_{\left(E_{\beta}\right)(\infty)} \neq 0$, then there exists a non-zero simple subcomodule $C^{*} x \subseteq M_{\left(E_{\alpha}\right)^{(\infty)}} \cap \sum_{\beta \in \bar{\Omega}, \beta \neq \alpha} M_{\left(E_{\beta}\right)^{(\infty)}}$. By [6, Proposition 1.16], $\langle x\rangle$ is a minimal closed subcomodule of $M$. By Lemma 1.1(3), $M_{\left(E_{\alpha}\right)^{(\infty)}}$ is a closed subcomodule of $M$. 
By [6, Lemma 3.3], there exists $\gamma \in \bar{\Omega}$ with $\gamma \neq \alpha$ such that $C^{*} x \subseteq M_{\left(E_{\gamma}\right)(\infty)}$. Thus $M_{\left(E_{\gamma}\right)(\infty)} \cap M_{\left(E_{\alpha}\right)(\infty)} \neq 0$. By Lemma 1.1 $(7),\left(E_{\gamma}\right)^{(\infty)} \cap\left(E_{\alpha}\right)^{(\infty)} \neq 0$, which contradicts [5]. Thus for any $\alpha \in \bar{\Omega}, M_{\left(E_{\alpha}\right)(\infty)} \cap \sum_{\beta \in \bar{\Omega}, \beta \neq \alpha} M_{\left(E_{\alpha}\right)(\infty)}=0$, which implies that

$$
M=\sum_{\alpha \in \bar{\Omega}} \oplus M_{\left(E_{\alpha}\right)(\infty)} .
$$

It follows from equations (2) and (3) that

$$
M_{\left(E_{\alpha}\right)(\infty)}=\sum_{i \in I_{\alpha}}\left(M_{E(\alpha, i)}\right)^{(\infty)}
$$

We see that

$$
\begin{aligned}
M_{E_{\alpha}} \wedge M_{E_{\alpha}} & \subseteq M_{E_{\alpha} \wedge E_{\alpha}} \quad \text { (by Lemma 1.6 (1)) } \\
& \subseteq M_{\left(E_{\alpha}\right)(\infty)} .
\end{aligned}
$$

Thus

$$
M_{\left(E_{\alpha}\right)^{(\infty)}} \supseteq\left(M_{E_{\alpha}}\right)^{(\infty)} \supseteq \sum_{i \in I_{\alpha}}\left(M_{E(\alpha, i)}\right)^{(\infty)}
$$

and

$$
M_{\left(E_{\alpha}\right)(\infty)}=\left(M_{E_{\alpha}}\right)^{(\infty)}
$$

by relation ( 4 ). This completes the proof.

Definition 1.9 If $M$ can be decomposed into a direct sum of two non-zero weak-closed subcomodules, then $M$ is called decomposable. If $N$ is a subcomodule of $M$ and $N$ contains exactly one non-zero minimal closed submodule, then $N$ is said to be relative-irreducible.

Corollary $1.10 C$ is a coalgebra.

(1) If $C$ is $\pi$-commutative, then every $C$-comodule $M$ is also $\pi$-commutative;

(2) If $C$ can be decomposed into a direct sum of its irreducible subcoalgebras, then every $C$-comodule $M$ can also be decomposed into a direct sum of its relative-irreducible subcomodules;

(3) If $C$ is decomposable, then every component faithfulness $C$-comodule $M$ is decomposable;

(4) If $C$ is irreducible, then every non-zero $C$-comodule $M$ is relative-irreducible;

(5) $C$ is irreducible iff every component faithfulness $C$-comodule $M$ is relative-irreducible.

Proof. (1) For any pair of non-zero closed subcomodules $N$ and $L$ of $M$, by Theorem 1.3, there exist faithful simple subcoalgebras $D$ and $E$ of $C$ to $M$ such that $N=M_{D}$ and $L=M_{E}$. By Lemma 1.6(2), $N \wedge L=M_{D} \wedge M_{E}=M_{D \wedge E}=M_{E \wedge D}=M_{E} \wedge M_{D}=L \wedge N$. Thus $M$ is $\pi$-commutative. 
(2) Since $C$ can be decomposed into a direct sum of its irreducible subcoalgebras, every equivalence class of $C$ contains only one element by [5]. By Theorem 1.8(1), every equivalence class of $M$ also contains only one element. Thus it follows from [6, Theorem 4.18] that $M$ can be decomposed into a direct sum of its relative-irreducible subcomodules.

(3) If $C$ is decomposable, then there are at least two non-zero equivalence classes in $C$. By Theorem 1.8(2), there are at least two non-zero equivalence classes in $M$. Thus $M$ is decomposable.

(4) If $C$ is irreducible, then there is only one non-zero simple subcoalgebra of $C$ and there is at most one non-zero minimal closed subcomodule in $M$ by Theorem 1.3. Considering $M \neq 0$, we have that $M$ is relative-irreducible.

(5) If $C$ is irreducible, then every component faithfulness $C$-comodule $M$ is relativeirreducible by Corollary 1.10(4). Conversely, let $M=C$ be the regular $C$-comodule. If $D$ is a non-zero simple subcoalgebra of $C$, then $0 \neq D \subseteq M_{D}$. Thus $M$ is a component faithfulness $C$-comodule. Since $M$ is a relative-irreducible $C$-comodule by assumption, there is only one non-zero minimal closed subcomodule in $M$ and so there is also only one non-zero simple subcoalgebra in $C$ by Theorem 1.3. Thus $C$ is irreducible.

Lemma 1.11 Let $N$ be a $C$-subcomodule of $M$ and $\emptyset \neq L \subseteq M$. Then

(1) $C^{*} \cdot L=C(M)^{*} \cdot L ; \quad N^{\perp C^{*} \perp M}=N^{\perp C(M)^{*} \perp M}$;

(2) $N$ is a (weak-) closed $C$-subcomodule iff $N$ is a (weak-)closed $C(M)$-subcomodule;

(3) $N$ is a minimal closed $C$-subcomodule iff $N$ is a minimal closed $C(M)$-subcomodule;

(4) $N$ is an indecomposable $C$-subcomodule iff $N$ is an indecomposable $C(M)$-subcomodule;

(5) $N$ is a relative-irreducible $C$-subcomodule iff $N$ is a relative-irreducible $C(M)$ subcomodule.

Proof. (1) Let $C=C(M) \oplus V$, where $V$ is a subspace of $C$. If $f \in V^{*}$, then $f \cdot L=0$. Thus $C^{*} \cdot L=\left(C(M)^{*}+V^{*}\right) \cdot L=C(M)^{*} \cdot L$. We now show the second equation. Obviously,

$$
N^{\perp C^{*} \perp M} \subseteq N^{\perp C(M)^{*} \perp M} .
$$

Conversely, for any $x \in N^{\perp C(M)^{*} \perp M}$ and $f \in N^{\perp C^{*}}$, there exist $f_{1} \in C(M)^{*}$ and $f_{2} \in V^{*}$ such that $f=f_{1}+f_{2}$. Obviously, $f \cdot x=f_{1} \cdot x=0$. Thus $x \in N^{\perp C^{*} \perp M}$ and $N^{\perp C(M)^{*} \perp M} \subseteq$ $N^{\perp C^{*} \perp M}$. Therefore

$$
N^{\perp C^{*} \perp M}=N^{\perp C(M)^{*} \perp M} .
$$

(2) If $N$ is a weak-closed $C$-subcomodule, then $\left(C^{*} \cdot x\right)^{\perp C^{*} \perp M} \subseteq N$ for any $x \in N$. We see that

$$
\begin{aligned}
\left(C^{*} \cdot x\right)^{\perp C^{*} \perp M} & =\left(C(M)^{*} \cdot x\right)^{\perp C^{*} \perp M}(\text { by Lemma } 1.11(1)) \\
& =\left(C(M)^{*} \cdot x\right)^{\perp C(M)^{*} \perp M} \quad(\text { by Lemma 1.11(1)) }
\end{aligned}
$$


Thus $\left(C(M)^{*} \cdot x\right)^{\perp C(M)^{*} \perp M} \subseteq N$ and so $N$ is a weak-closed $C(M)$-subcomodule. Conversely, if $N$ is a weak-closed $C(M)$-subcomodule. Similarly, we can show that $N$ is a weak-closed $C$-subcomodule. We now show the second assertion. If $N$ is a closed $C$-subcomodule, then $N^{\perp C^{*} \perp M}=N$ and $N=N^{\perp C^{*} \perp M}=N^{\perp C(M)^{*} \perp M}$ by part (1), which implies that $N$ is a closed $C(M)$-subcomodule. Conversely, if $N$ is a closed $C(M)$ subcomodule, similarly, we can show that $N$ is a closed $C$-subcomodule.

Similarly the others can be proved.

Proposition 1.12 Let every simple subcoalgebra in $C(M)$ be faithful to $M$.

(1) If $M$ is an indecomposable $C$-comodule, then $C(M)$ is also an indecomposable subcoalgebra.

(2) $M$ is a relative-irreducible $C$-comodule iff $C(M)$ is an irreducible subcoalgebra.

Proof. (1) If $M$ is an indecomposable $C$-comodule, then $M$ is an indecomposable $C(M)$ comodule by Lemma 1.11 and $M$ is a component faithfulness $C(M)$-comodule. By Corollary $1.10(3), C(M)$ is indecomposable.

(2) If $M$ is a relative-irreducible $C$-comodule, then $M$ is a relative-irreducible $C(M)$ comodule by Lemma $1.11(5)$ and $C(M)$ is irreducible. Conversely, if $C(M)$ is irreducible, then $M$ is a relative-irreducible $C(M)$-comodule by Corollary $1.10(4)$ and so $M$ is a relative-irreducible $C$-comodule by Lemma $1.11(5)$.

Definition 1.13 If $D \sim E$ implies $M_{D} \sim M_{E}$ for any simple subcoalgebras $D$ and $E$ in $C(M)$, then $M$ is called a $W$-relational hereditary $C$-comodule.

If $M$ is a $W$-relational hereditary $C$-comodule, then $\mathcal{C}(M)_{1}=\mathcal{C}(M)_{0}$. In fact, by Lemma 1.1(5), $\mathcal{C}(M)_{1} \subseteq \mathcal{C}(M)_{0}$. If $\mathcal{C}(M)_{1} \neq \mathcal{C}(M)_{0}$, then there exists $0 \neq D \in \mathcal{C}(M)_{0}$ such that $M_{D}=0$. Since $M_{D} \sim M_{0}$ and $M$ is $W$-relational hereditary, we have that $D \sim 0$ and $D=0$ by Lemma $1.7(1)$. We get a contradiction. Thus $\mathcal{C}(M)_{1}=\mathcal{C}(M)_{0}$.

Obviously, every $\pi$-commutative comodule is $W$-relational hereditary. If $C$ is $\pi$ commutative, then $M$ is $\pi$-commutative by Corollary $1.10(1)$ and every $C$-comodule $M$ is $W$-relational hereditary. Furthermore, $M$ is also a component faithfulness $C(M)$ comodule.

Proposition 1.14 Let the notation be the same as in Theorem 1.8. Then the following conditions are equivalent.

(1) $M$ is $W$-relational hereditary.

(2) For any $\alpha \in \bar{\Omega}$, there is at most one non-zero equivalence class in $\left\{M_{\mathcal{E}(\alpha, i)} \mid i \in I_{\alpha}\right\}$, and $\mathcal{C}(M)_{1}=\mathcal{C}(M)_{0}$.

(3) For any $D \in \mathcal{C}(M)_{0}$,

$\left[M_{D}\right]=\left\{M_{F} \mid F \in[D]\right.$ and $\left.F \subseteq C(M)\right\}$ 
(4) For any $D$ and $E \in \mathcal{C}(M)_{0}, M_{D} \sim M_{E}$ iff $D \sim E$.

(5) For any $\alpha \in \bar{\Omega}, M_{\left(E_{\alpha}\right)(\infty)}$ is indecomposable, and $\mathcal{C}(M)_{1}=\mathcal{C}(M)_{0}$.

(6) For any $\alpha \in \bar{\Omega},\left(M_{E_{\alpha}}\right)^{(\infty)}$ is indecomposable, and $\mathcal{C}(M)_{1}=\mathcal{C}(M)_{0}$;

(7) $M=\sum_{\alpha \in \bar{\Omega}} \oplus\left(M_{E_{\alpha}}\right)^{(\infty)}$ is its weak-closed indecomposable decomposition, and $\mathcal{C}(M)_{1}=$ $\mathcal{C}(M)_{0}$

(8) $M=\sum_{\alpha \in \bar{\Omega}} \oplus M_{\left(E_{\alpha}\right)(\infty)}$ is its weak-closed indecomposable decomposition, and $\mathcal{C}(M)_{1}=$ $\mathcal{C}(M)_{0}$.

Proof. We prove it along with the following lines: $(1) \Longrightarrow(4) \Longrightarrow(3) \Longrightarrow(1)(3) \Longleftrightarrow$ $(2) \Longleftrightarrow(5) \Longleftrightarrow(6) \Longleftrightarrow(7) \Longleftrightarrow(8)$.

$(1) \Longrightarrow(4)$ It follows from the discussion above that

$$
\mathcal{C}(M)_{1}=\mathcal{C}(M)_{0}
$$

If $D$ and $E \in \mathcal{C}(M)_{0}$ and $M_{D} \sim M_{E}$, then $D \sim E$ by Lemma 1.7 (2). If $D \sim E$ and $D$ and $E \in \mathcal{C}(M)_{0}$, then $M_{D} \sim M_{E}$.

$(4) \Longrightarrow(3)$ Considering Lemma $1.7(4)$ and $\mathcal{C}(M)_{1}=\mathcal{C}(M)_{0}$, we only need to show that

$$
\left\{M_{F} \mid F \in[D] \text { and } F \subseteq C(M)\right\} \subseteq\left[M_{D}\right]
$$

For any $F \in[D]$ with $F \subseteq C(M), F \sim D$ and $M_{F} \sim M_{D}$ by part (4), which implies that $M_{F} \in\left[M_{D}\right]$.

$(3) \Longrightarrow(1)$ It is trivial.

$(5) \Longleftrightarrow(6) \Longleftrightarrow(7) \Longleftrightarrow(8)$ It follows from Theorem $1.8(3)$.

$(5) \Longleftrightarrow(2)$ By Theorem 1.8 (3),

$$
M_{\left(E_{\alpha}\right)(\infty)}=\sum_{i \in I_{\alpha}}\left(M_{E(\alpha, i)}\right)^{(\infty)}
$$

Thus part (2) and part (5) are equivalent.

$(2) \Longrightarrow(3)$ It follows from Lemma 1.7 (4).

$(3) \Longrightarrow(2)$ If there are two non-zero equivalence classes $M_{\mathcal{E}\left(\alpha, i_{1}\right)} \neq 0$ and $M_{\mathcal{E}\left(\alpha, i_{2}\right)} \neq 0$ in $\left\{M_{\mathcal{E}(\alpha, i)} \mid i \in I_{\alpha}\right\}$, then there exist $D_{1} \in \mathcal{E}\left(\alpha, i_{1}\right)$ and $D_{2} \in \mathcal{E}\left(\alpha, i_{2}\right)$ such that $M_{D_{1}} \neq 0$ and $M_{D_{2}} \neq 0$. Let $D=D_{1}$. Since $M_{D_{1}}$ and $M_{D_{2}} \in\left\{M_{F} \mid F \in[D]\right.$ and $\left.F \subseteq C(M)\right\}$ and $M_{D_{2}} \notin\left[M_{D}\right]=M_{\mathcal{E}\left(\alpha, i_{1}\right)}$, this contradicts part (3). Thus there is at most one non-zero equivalence class in $\left\{M_{\mathcal{E}(\alpha, i)} \mid i \in I_{\alpha}\right\}$.

Proposition 1.15 If $M$ is a full, $W$-relational hereditary $C$-comodule, then

(1) $M$ is indecomposable iff $C$ is indecomposable;

(2) $M$ is relative-irreducible iff $C$ is irreducible.

(3) $M$ can be decomposed into a direct sum of its weak-closed relative-irreducible subcomodules iff $C$ can be decomposed into a direct sum of its irreducible subcoalgebras.

(4) $M$ is $\pi$-commutative iff $C$ is $\pi$-commutative. 
Proof. Since $M$ is a full $C$-comodule, $C(M)=C$. Since $M$ is $W$-relational hereditary, $\mathcal{C}(M)_{1}=\mathcal{C}(M)_{0}=\mathcal{C}_{0}$. Thus $M$ is a component faithfulness $C$-comodule.

(1) If $M$ is indecomposable, then $C$ is indecomposable by Proposition 1.12(1). Conversely, if $C$ is indecomposable, then there is at most one non-zero equivalence class in $C$. By Proposition 1.14(2), there is at most one non-zero equivalence class in $M$. Thus $M$ is indecomposable.

(4) If $M$ is $\pi$-commutative, then there is only one element in every equivalence class of $M$. By Proposition 1.14(4) and Theorem 1.3, there is only one element in every equivalence class of $C$. Thus $C$ is $\pi$-commutative by [5]. Conversely, if $C$ is $\pi$-commutative, then $M$ is $\pi$-commutative by Corollary $1.10(1)$.

(2) It follows from the above discussion and Proposition 1.12.

$(3) \Longleftrightarrow(4)$ By [6, Theorem3.8 and Theorem 4.18] and [5], it is easy to check that (3) and (4) are equivalent.

Proposition 1.16 If $M_{D} \wedge M_{E}=M_{E} \wedge M_{D}$ implies $D \wedge E=E \wedge D$ for any simple coalgebras $D$ and $E$ in $C(M)$, then $M$ is $W$-relational hereditary.

Proof. Let $D \sim E$. For any pair of subclasses $\mathcal{M}_{1}$ and $\mathcal{M}_{2}$ of $\mathcal{M}_{0}$ with $\mathcal{M}_{1} \cap \mathcal{M}_{2}=\emptyset$ and $\mathcal{M}_{1} \cup \mathcal{M}_{2}=\mathcal{M}_{0}$, if $M_{D} \in \mathcal{M}_{1}$ and $M_{E} \in \mathcal{M}_{2}$, let $\mathcal{C}_{1}=\left\{F \in \mathcal{C}_{0} \mid M_{F} \in \mathcal{M}_{1}\right\}$ and $\mathcal{C}_{2}=\left\{F \in \mathcal{C}_{0} \mid M_{F} \in \mathcal{M}_{2}\right\}$. By Theorem 1.3, $\mathcal{C}_{0}=\mathcal{C}_{1} \cup \mathcal{C}_{2}$ and $\mathcal{C}_{1} \cap \mathcal{C}_{2}=\emptyset$. Obviously, $D \in \mathcal{C}_{1}$ and $E \in \mathcal{C}_{2}$. By Definition 1.4, there exist $D_{1} \in \mathcal{C}_{1}$ and $E_{1} \in \mathcal{C}_{2}$ such that $D_{1} \wedge E_{1} \neq E_{1} \wedge D_{1}$. By the assumption condition, we have that $M_{D_{1}} \wedge M_{E_{1}} \neq M_{E_{1}} \wedge M_{D_{1}}$. Thus $M_{D} \sim M_{E}$, i.e. $M$ is $W$-relational hereditary.

Proposition 1.17 Let $M=C$ as a right $C$-comodule. Let $N=D$ and $L=E$ with $N$ and $L$ as subcomodules of $M$ with $D$ and $E$ as right coideals of $C$. Let $X$ be an ideal of $C^{*}$ and $F$ be a subcoalgebra of $C$. Then:

(1) $X^{\perp C}=X^{\perp M} ; \quad N^{\perp C^{*} \perp M}=C(N) ; \quad C\left(M_{F}\right)=F$;

(2) $N$ is a closed subcomodule of $M$ iff $D$ is a subcoalgebra of $C$;

(3) $N$ is a closed subcomodule iff $N$ is a weak-closed subcomodule of $M$;

(4) $N$ is a minimal closed subcomodule of $M$ iff $D$ is a simple subcoalgebra of $C$;

(5) When $N$ and $L$ are closed subcomodules, $N \wedge_{M} L=D \wedge_{C} E$, where $\wedge_{M}$ and $\wedge_{C}$ denote wedge in comodule $M$ and in coalgebra $C$ respectively;

(6) $M$ is a full and $W$-relational hereditary $C$-comodule and a component faithfulness C-comodule.

(7) The weak-closed indecomposable decomposition of $M$ as a $C$-comodule and the indecomposable decomposition of $C$ as coalgebra are the same.

Proof. (1) If $x \in X^{\perp M}$, then $f \cdot x=0$ for any $f \in X$. Let $\rho(x)=\sum_{i=1}^{n} x_{i} \otimes c_{i}$ and $x_{1}$, $\cdots, x_{n}$ be linearly independent. Thus $f\left(c_{i}\right)=0$ and $i=1, \cdots, n$. Since $x=\sum_{i=1}^{n} \epsilon\left(x_{i}\right) c_{i}$, $f(x)=0$, which implies $x \in X^{\perp C}$. 
Conversely, if $x \in X^{\perp C}$, we have that $\rho(x) \in X^{\perp C} \otimes X^{\perp C}$ since $X^{\perp C}$ is subcoalgebra of $C$. Thus $f \cdot x=0$ for any $f \in X$, which implies $x \in X^{\perp M}$. Thus $X^{\perp M}=X^{\perp C}$. By Lemma 1.1(1), $N^{\perp C^{*} \perp M}=C(N)^{\perp C^{*} \perp M}$. By part (1), $C(N)^{\perp C^{*} \perp M}=C(N)^{\perp C^{*} \perp C}$. Thus $N^{\perp C^{*} \perp M}=C(N)^{\perp C^{*} \perp C}=C(N)$.

Finally, we show that $C\left(M_{F}\right)=F$. Obviously, $C\left(M_{F}\right) \subseteq F$. If we view $F$ as a $C$-subcomodule of $M$, then $F \subseteq C\left(M_{F}\right)$. Thus $F=C\left(M_{F}\right)$.

(2) If $N$ is a closed subcomodule of $M$, then $N^{\perp C^{*} \perp M}=N$. By Proposition 1.17(1), $N^{\perp C^{*} \perp M}=C(N)=N$. Thus $\rho(N)=\Delta(D) \subseteq N \otimes N=D \otimes D$, which implies that $D$ is a subcoalgebra of $C$. Conversely, if $D$ is a subcoalgebra of $C$, then $\rho(N) \subseteq N \otimes N$. Thus $C(N) \subseteq D=N$. By Proposition 1.17(1), $N^{\perp C^{*} \perp M}=C(N) \subseteq N$. Thus $N^{\perp C^{*} \perp M}=N$, i.e. $N$ is closed.

(3) If $N$ is a closed subcomodule, then $N$ is weak-closed. Conversely, if $N$ is weakclosed, then $\langle x\rangle \subseteq N$ for $x \in N$. Since $\langle x\rangle$ is a closed subcomodule of $M$, $\langle x\rangle$ is subcoalgebra of $C$ if let $\langle x\rangle$ with structure of coalgebra $C$. This shows that $\Delta(x) \in\langle x\rangle \otimes\langle x\rangle \subseteq D \otimes D$. Thus $D$ is a subcoalgebra of $C$. By Proposition 1.17(2), $N$ is a closed subcomodule of $M$.

(4) It follows from part (2).

(5) We only need to show that

$$
\left(N^{\perp C^{*}} L^{\perp C^{*}}\right)^{\perp M}=\left(D^{\perp C^{*}} E^{\perp C^{*}}\right)^{\perp C} .
$$

Since $N$ and $L$ are closed subcomodules, $N^{\perp C^{*}}=C(N)^{\perp C^{*}}=D^{\perp C^{*}}$ and $L^{\perp C^{*}}=$ $C(L)^{\perp C^{*}}=E^{\perp C^{*}}$ by Proposition 1.17(1) and Lemma 1.1(1). Thus we only need to show that

$$
\left(D^{\perp C^{*}} E^{\perp C^{*}}\right)^{\perp M}=\left(D^{\perp C^{*}} E^{\perp C^{*}}\right)^{\perp C} .
$$

The above formula follows from Proposition 1.17(1).

(6) By the proof of Corollary 1.10(5), we know that $M$ is a component faithfulness $C$-comodule. Let $\left\{m_{\lambda} \mid \lambda \in \Lambda\right\}$ be a basis of $M$. For any $c \in C, \Delta(c)=\sum m_{\lambda} \otimes c_{\lambda}$, by the definition of $C(M), c_{\lambda} \in C(M)$. Since $c=\sum \epsilon\left(m_{\lambda}\right) c_{\lambda} \in C(M), C \subseteq C(M)$, i.e. $C=C(M)$. Consequently, it follows from part (4)(5) that $M$ is $W$-relational hereditary.

(7) Since $M$ is $W$-relational hereditary, $M=\sum_{\alpha \in \bar{\Omega}} \oplus M_{\left(E_{\alpha}\right)(\infty)}$ is a weak-closed indecomposable decomposition of $M$ by Proposition 1.14. By part (1) (3), $C\left(M_{\left(E_{\alpha}\right)(\infty)}\right)=$ $\left(E_{\alpha}\right)^{(\infty)}=\left(M_{\left(E_{\alpha}\right)(\infty)}\right)^{\perp C^{*} \perp M}=M_{\left(E_{\alpha}\right)(\infty)}$. Thus $M=\sum_{\alpha \in \bar{\Omega}} \oplus M_{\left(E_{\alpha}\right)(\infty)}=\sum_{\alpha \in \bar{\Omega}} \oplus\left(E_{\alpha}\right)^{(\infty)}$. By [5], $\sum_{\alpha \in \bar{\Omega}} \oplus\left(E_{\alpha}\right)^{(\infty)}=C$ is a indecomposable decomposition of $C$. Thus the weakclosed indecomposable decomposition of $M$ as a $C$-comodule and the indecomposable decomposition of $C$ as coalgebra are the same. This completes the proof.

By Lemma 1.6(4) and Proposition 1.17, $C$ is cosemisimple iff every $C$-comodule $M$ is cosemisimple. 


\section{The coradicals of comodules}

Proposition 2.1 Let $M$ be a $C$-comodule, $J$ denote the Jacobson radical of $C^{*}$ and $r_{j}\left(C(M)^{*}\right)$ denote the Jacobson radical of $C(M)^{*}$.

(1)

$$
M_{0}=\left(r_{j}\left(C(M)^{*}\right)\right)^{\perp M}=\operatorname{Soc}_{C^{*}} M \quad \text { and } \quad r_{j}\left(C(M)^{*}\right)=M_{0}^{\perp C^{*}}
$$

(2) If we view $C$ as a right $C$-comodule, then

$$
C_{0}=S_{o c} C^{*} C=J^{\perp C}=\sum\{D \mid D \text { is a minimal right coideal of } C\} .
$$

Proof. (1) We first show that

$$
M_{0}^{\perp C^{*}} \subseteq J
$$

when $M$ is a full $C$-comodule. We only need to show that $\epsilon-f$ is invertible in $C^{*}$ for any $f \in\left(M_{0}\right)^{\perp C^{*}}$. Let $I=\left(M_{0}\right)^{\perp C^{*}}$. For any natural number $n, f^{n+1} \cdot\left(M_{0}\right)^{(n)}=0$ since $f^{n+1} \in I^{n+1}$, where $\left(M_{0}\right)^{(n)}=\wedge^{n+1} M_{0}$. Thus

$$
f^{n+1}\left(C\left(\left(M_{0}\right)^{(n)}\right)\right)=0 .
$$

by Lemma 1.1(1) and

$$
M=\left(M_{0}\right)^{(\infty)}=\cup\left\{\left(M_{0}\right)^{(n)} \mid n=0,1, \cdots\right\}
$$

by $[6$, Theorem 4.7]. We now show that

$$
C=\cup\left\{C\left(\left(M_{0}\right)^{(n)}\right) \mid n=0,1, \cdots\right\}
$$

Since $\left(M_{0}\right)^{(n)} \subseteq\left(M_{0}\right)^{(n+1)}$, we have that there exists a basis $\left\{m_{\lambda} \mid \lambda \in \Lambda\right\}$ of $M$ such that for every given natural number $n$ there exists a subset of $\left\{m_{\lambda} \mid \lambda \in \Lambda\right\}$, which is a basis of $\left(M_{0}\right)^{(n)}$. For any $c \in W(M)$, there exists $m \in M$ with $\rho(m)=\sum m_{\lambda} \otimes c_{\lambda}$ such that $c_{\lambda_{0}}=c$ for some $\lambda_{0} \in \Lambda$. By equation (6), there exists a natural number $n$ such that $m \in\left(M_{0}\right)^{(n)}$, which implies that $c \in C\left(\left(M_{0}\right)^{(n)}\right)$ and $C(M) \subseteq \cup_{0}^{\infty} C\left(\left(M_{0}\right)^{(n)}\right)$. Thus

$$
C=\cup\left\{C\left(\left(M_{0}\right)^{(n)}\right) \mid n=0,1, \cdots\right\}
$$

Let

$$
g_{n}=\epsilon+f+\cdots+f^{n}, n=1,2, \cdots .
$$

For any $c \in C$, there exists a natural number $n$ such that $c \in C\left(\left(M_{0}\right)^{(n)}\right)$ by relation (7). We define

$$
g(c)=g_{n}(c) .
$$


Considering relation (5), we have that $g$ is well-defined. Thus $g \in C^{*}$.

We next show that $g$ is an inverse of $\epsilon-f$ in $C^{*}$. For any $c \in C^{*}$, there exists a natural number $n$ such that $c \in C\left(\left(M_{0}\right)^{(n)}\right)$ by relation (7). Thus $\Delta(c) \in C\left(\left(M_{0}\right)^{(n)}\right) \otimes C\left(\left(M_{0}\right)^{(n)}\right)$. We see that

$$
\begin{aligned}
g *(\epsilon-f)(c) & =\sum g\left(c_{1}\right)(\epsilon-f)\left(c_{2}\right) \\
& \left.=\sum g_{n}\left(c_{1}\right)(\epsilon-f)\left(c_{2}\right) \text { (by relation }(8)\right) \\
& =\left(g_{n} *(\epsilon-f)\right)(c) \\
& =\left(\epsilon-f^{n+1}\right)(c) \\
& =\epsilon(c)(\text { by relation }(5)) .
\end{aligned}
$$

Thus $g *(\epsilon-f)=\epsilon$. Similarly, $(\epsilon-f) * g=\epsilon$. Thus $\epsilon-f$ has an inverse in $C^{*}$.

We next show that

$$
M_{0}^{\perp C^{*}}=J
$$

when $C(M)=C$. It follows from Lemma 1.1(1) that

$$
\begin{aligned}
\left(M_{0}\right)^{\perp C^{*}} & =\cap\left\{N^{\perp C^{*}} \mid N \text { is a minimal closed subcomodule of } M\right\} \\
& =\cap\left\{C(N)^{\perp C^{*}} \mid N \text { is a minimal closed subcomodule of } M\right\} \\
& \supseteq J(\text { by }[2, \text { Proposition 2.1.4] and Proposition 1.2) }
\end{aligned}
$$

Thus $M_{0}^{\perp C^{*}}=J$

We now show that

$$
r_{j}\left(C(M)^{*}\right)=M_{0}^{\perp C^{*}} \quad \text { and } \quad\left(r_{j}\left(C(M)^{*}\right)\right)^{\perp M}=M_{0}
$$

for any $C$-comodule $M$. If $M$ is a $C$-comodule, then $M$ is full $C(M)$-comodule and so

$$
\left(r_{j}\left(C(M)^{*}\right)=M_{0}^{\perp C^{*}}\right.
$$

By [6, Proposition 4.6], $M_{0}$ is closed. Thus $\left(\left(r_{j}\left(C(M)^{*}\right)\right)^{\perp M}=M_{0}\right.$.

Finally, we show that $M_{0}=\operatorname{Soc}_{C^{*}} M$ for any $C$-comodule $M$. By [6, Proposition 1.16], $S_{o c} C^{*} M \subseteq M_{0}$. Conversely, if $x \in M_{0}$, let $N=C^{*} x$. By Lemma 1.6(4), $M_{C_{0}}=M_{0}$. Thus $N$ is a finite dimensional $C_{0}$-comodule. By [4, Theorem14.0.1], $N=N_{1} \oplus \cdots \oplus N_{n}$ and $N_{i}$ is a simple $C_{0}^{*}$-submodule. By [6, Lemma 1.1], $N_{i}$ is a $C_{0}$-subcomodule. Thus $N_{i}$ is a $C$-comodule. By [6, Lemma 1.1], $N_{i}$ is a $C^{*}$-submodule. If $L$ is a non-zero $C^{*}$-submodule of $M$ and $L \subseteq N_{i}$, then $L$ is also a $\left(C_{0}\right)^{*}$-submodule. Thus $L=N_{i}$. This shows that $N_{i}$ is also a simple $C^{*}$-submodule. Thus $N_{i} \subseteq \operatorname{Soc}_{C^{*}} M$ and $N \subseteq \operatorname{Soc}_{C^{*}} M$. It follows from the above proof that $M_{0}=S_{C_{C}} M$.

(2) It follows from Proposition 1.17 and part (1). 


\section{References}

[1] E. Abe. Hopf Algebra. Cambridge University Press, 1980.

[2] R. G. Heyneman and D. E. Radford. Reflexivity and coalgebras of finite type. J. Algebra 28 (1974), 215-246.

[3] T. Shudo And H. Miyamito. On the decomposition of coalgebras. Hiroshima Math. J., 8(1978), 499-504.

[4] M. E. Sweedler. Hopf Algebras. Benjamin, New York, 1969.

[5] Y. H. Xu and Y. Fong. On the decomposition of coalgebras. In "Proceedings International Colloquium on Words, Languages and Combinatorics- Kyoto,August 28-31, 1990". pp. 504-522, World Scientific , Singapore, 1992.

[6] Y. H. Xu, K. P. Shum and Y. Fong. A decomposition Theory of Comodules. J. Algebra, 170(1994), 880-896.

[7] X. G. Zou and Y. H. Xu. The decomposition of comodules. Science in China (Series A), 37(1994)8, 946-953. 